\title{
A specific structural alteration in the heparan sulphate of human glomerular basement membrane in diabetes
}

\author{
A.S.B.Edge, R. G.Spiro \\ Departments of Biological Chemistry and Medicine, Harvard Medical School and the Joslin Diabetes Center, Boston, Mass., USA
}

\section{Abstract}

Aims/hypothesis. Heparan sulphate proteoglycan is an important component of the glomerular anionic filtration barrier and its reduced amount in diabetes contributes to glomerular dysfunction. The objective of this study was to determine if there is also an alteration in the sulphation pattern of the diabetic heparan sulphate chains.

Methods. The heparan sulphate in the glomerular basement membrane/mesangial matrix from human diabetic and nondiabetic kidneys obtained at autopsy was fragmented by a hydrazine/nitrous acid procedure and after radiolabelling with $\mathrm{NaB}\left[{ }^{3} \mathrm{H}\right]_{4}$, the disaccharide products were chromatographically resolved and quantified.

Results. Six sulphated disaccharides were identified in both the diabetic and nondiabetic samples and the molar distribution of these was similar, with the notable exception of the iduronic acid-2-O-sulphate $\alpha 1 \rightarrow$ 4glucosamine-3-O-sulphate species which occurred in the diabetic glomeruli in less than half the amount as in the nondiabetic samples $(9.0 \%$ compared to $18.7 \%$ of total sulphated disaccharides, $p<0.005$ ).
Conclusion/interpretation. 3-O-sulphated glucosamine is a rare constituent of heparan sulphate occurring usually in a glucuronic acid $\beta 1 \rightarrow 4$ glucosamine-3-O-sulphate( $\pm 6-O$-sulphate $)$ sequence within the antithrombin-binding domain. In the glomerular basement membrane where the 3-O-sulphated glucosamine is present in substantial amounts, however, it occurs exclusively in an iduronic acid-containing sequence. It is likely that the recently discovered 3-O-sulphotransferase variant which specifically acts on the iduronic acid $\alpha 1 \rightarrow 4$ glucosamine sequence is decreased in human diabetes and moreover that this unusual disaccharide could be a component of a specific heparan sulphate domain which interacts with bioactive proteins. [Diabetologia (2000) 43: 1056-1059]

Keywords Glomerular basement membrane, diabetic glomerular basement membrane alterations, 3- $O$-sulphated glucosamine, heparan sulphate binding, heparan sulphate sulphation sites, hydrazine/nitrous acid fragmentation, iduronic acid, glomerular anionic filter.
The alterations affecting the macromolecules of the glomerular basement membrane and adjoining mesangium (GBM) are believed to have a major role in

Corresponding author: Dr. R. G. Spiro, Joslin Diabetes Center, One Joslin Place, Boston, Mass. 02215, USA

Abbreviations: GBM, glomerular basement membrane and adjoining mesangium; GlcN, D-glucosamine; GlcUA, D-glucuronic acid; IdUA, L-iduronic acid; $\mathrm{AnManH}_{2}$, 2,5-anhydro-Dmannitol; NS, N-sulphate; $2 \mathrm{~S}, 2-O$-sulphate; 3S, 3-O-sulphate; 6S, 6- $O$-sulphate the glomerular dysfunction of diabetes. Both collagenous and noncollagenous components of the glomerular filtration barrier have been shown to undergo biochemical derangements in this disease which contribute to the renal pathophysiology [1-3].

Of particular interest in this regard are changes in the GBM heparan sulphate which serves as an important anionic component of the renal filtration barrier [3]; both biochemical and immunochemical measurements of the heparan sulphate proteoglycan and its glycosaminoglycan chains have shown a reduction in 
the human diabetic kidney $[2,4]$ and decreased $N$ deacetylation $/ N$-sulfation has been noted in the diabetic rat [3].

Procedures to fragment glycosaminoglycans has made it possible to identify and quantify the diverse sulphated disaccharide units and this approach has already provided valuable information in regard to the fine structure of the bovine GBM heparan sulphate [5]. In this study we have applied the hydrazine/nitrous acid $/ \mathrm{NaB}\left[{ }^{3} \mathrm{H}_{4}\right]$ cleavage technique to the GBM of human diabetic and nondiabetic subjects to determine whether alterations in the heparan sulphate sequence or sulphation pattern or both occur in the disease state. These analyses showed that the diabetic GBM contained one specific structural unit, namely IdUA(2S) $\alpha 1 \rightarrow 4 \mathrm{GlcN}(3 \mathrm{~S})$, in significantly decreased amounts. This particular disaccharide, which has previously been found in the bovine GBM [5], is of interest in that its occurrence seems to be limited to GBM heparan sulphate proteoglycans and furthermore, 3$O$-sulphated glucosamine, which is a rare constitutent of heparan sulphate chains, has been implicated as a component of the antithrombin-binding region $[6,7]$. Moreover, a recent report [8] has shown the occurrence of a specific glucosaminyl 3-O-sulphotransferase which is involved in the generation of the IdUA(2S) $\alpha 1 \rightarrow 4 \mathrm{GlcN}(3 \mathrm{~S})$, suggesting that this sequence in the GBM could have a distinct biological function.

\section{Subjects, materials and methods}

Preparation of GBM. Human kidneys were obtained at autopsy (New England Deaconess and the Brigham and Women's Hospitals) from diabetic and nondiabetic patients and stored at $-20^{\circ} \mathrm{C}$ until GBM was prepared as described previously [4]. Individual kidneys used for the preparation of GBM were from eight nondiabetic and six diabetic subjects whose ages ranged for the nondiabetic from 56 to 78 years (means \pm SEM $=64.4 \pm 7.2$ ) and for diabetic from 52 to 83 years (means \pm SEM $=69.0 \pm 6.4$ ), respectively. All of the diabetic patients had been on insulin therapy and the duration of their disease ranged from 10 to 30 years (means \pm SEM $=20 \pm 5.8$ ). The diabetic kidneys varied in glomerulopathy while the nondiabetic kidneys were histologically normal. The yield of GBM from the diabetic kidneys was higher (means $\pm \mathrm{SEM}=30.7 \pm 10 \mathrm{mg} / 100 \mathrm{~g}$ cortex $)$ than from the normal kidneys (means $\pm \mathrm{SEM}=17.3 \pm 2.6 \mathrm{mg} / 100 \mathrm{~g}$ cortex). A large-scale preparation of normal GBM was prepared from the pooled glomeruli of several nondiabetic subjects for initial characterisation studies.

Estimation of heparan sulphate proteoglycan by immunoassay. The amount of proteoglycan present in GBM from individual cases was determined by a sol- id phase radioimmunoassay employing an antibody raised against the bovine heparan sulphate proteogycan [4].

Fragmentation of glycosaminoglycan chains followed by the $\mathrm{NaB}\left[{ }^{3} \mathrm{H}\right]_{4}$ reduction of saccharide products. Isolated GBM samples ( 1.5 to $2 \mathrm{mg}$ ) were treated by the hydrazine/nitrous acid procedure to cleave hexosaminidic bonds as described previously [5]. Subsequently, the desalinated disaccharide products were reduced with $37 \mathrm{MBq} \mathrm{NaB}\left[{ }^{3} \mathrm{H}_{4}\right](377 \mathrm{GBq} / \mathrm{mmol}$, DuPont-New England Nuclear, Boston, Mass., USA) [5]; an $\mathrm{N}$-acetylglucosamine standard was reduced concurrently. In this fragmentation procedure disaccharides containing hexosamines yield anhydromannitol and anhydrotalitol from glucosamine and galactosamine, respectively.

Separation and quantification of radiolabelled disaccharides. Fractionation and resolution of the disaccharides was achieved by sequential Dowex 1 (BioRad), DEAE-cellulose and thin layer chromatography, in a manner detailed previously [5]. To identify the purified components, radiolabelled disaccharide standards were prepared from bovine GBM, heparin and heparin oligosaccharides [5]. Quantitation of the purified radioactive disaccharides, visualised by fluorography on thin layer chromatographic plates, was achieved by scintillation counting. The protein content of GBM samples was determined from amino acid analyses.

\section{Results}

Immunoassays carried out on the GBM from the individual cases indicated a reduction in the amount of heparan sulphate proteoglycan in the diabetic samples $(41 \%$ of nondiabetic; $p<0.002)$, which was consistent with that previously reported for a different group of cases [4].

Treatment of GBM with hydrazine followed by nitrous acid to bring about cleavage of the glycosaminoglycans at the hexosamine residue produced disaccharides which after ion exchange column chromatography were identified by thin layer chromatography. While the nonsulfated uronic acid-containing disaccharide fraction, which contained predominantly GlcUA $\beta 1 \rightarrow 4 \mathrm{AnManH}_{2}$, represented $76 \%$ and $73 \%$ of the total disaccharide radioactivity in the nondiabetic and diabetic samples respectively, the remaining disaccharides were distributed among six sulphated species containing anhydromannitol (Table 1) characteristic of heparan sulphate. These components were the same as previously identified in the bovine GBM [5].

A comparison of the molar distribution of the sulphated disaccharide units in the nondiabetic and dia- 
Table 1. Quantification of the heparan sulphate disaccharides released from normal and diabetic human GBM by hydrazine/nitrous acid fragmentation ${ }^{1}$

\begin{tabular}{|c|c|c|c|}
\hline Disaccharide $^{2}$ & Nondiabetic & Diabetic & $p$ value \\
\hline & \multicolumn{3}{|c|}{ Per cent of total sulphated disaccharides ${ }^{3}$} \\
\hline $\mathrm{GlcUA}_{\beta} 1 \rightarrow 4 \mathrm{AnManH}_{2}(6 \mathrm{~S})$ & $21.1 \pm 1.2$ & $23.6 \pm 3.3$ & $\mathrm{NS}^{4}$ \\
\hline $\mathrm{IdUA}_{\alpha 1} \rightarrow 4 \mathrm{AnManH}_{2}(3 \mathrm{~S})$ & $16.8 \pm 2.8$ & $18.6 \pm 2.8$ & NS \\
\hline $\mathrm{IdUA}(2 \mathrm{~S}) \alpha 1 \rightarrow 4 \mathrm{AnManH}_{2}$ & $21.4 \pm 2.2$ & $27.7 \pm 5.0$ & NS \\
\hline IdUA $\alpha 1 \rightarrow 4 \mathrm{AnManH}_{2}(6 \mathrm{~S})$ & $12.6 \pm 0.8$ & $15.0 \pm 2.0$ & NS \\
\hline $\operatorname{IdUA}(2 \mathrm{~S}) \alpha 1 \rightarrow 4 \mathrm{AnManH}_{2}(3 \mathrm{~S})$ & $18.7 \pm 2.2$ & $9.0 \pm 1.5$ & $<0.005$ \\
\hline $\operatorname{IdUA}(2 \mathrm{~S}) \alpha 1 \rightarrow 4 \mathrm{AnManH}_{2}(6 \mathrm{~S})$ & $10.2 \pm 2.6$ & $6.7 \pm 1.1$ & NS \\
\hline
\end{tabular}

\begin{abstract}
${ }^{1}$ After hydrazine nitrous acid treatment of the GBM, the released disaccharides were reduced with $\mathrm{NaB}\left[{ }^{3} \mathrm{H}\right]_{4}$ and fractionated by Dowex 1 and DEAE-cellulose chromatography. The total yield of uronic acid-containing disaccharides was $1.96 \pm 0.30$ and $2.89 \pm 0.38 \mathrm{nmol} / \mathrm{mg} \mathrm{GBM}$ in the diabetic and nondiabetic samples respectively. The sulphated disaccharides were further resolved by thin layer chromatography and identified by comparison to the migration of disaccharide standards. Quantification of the disaccharides was achieved by scintillation counting after elution from the chromatogram.

${ }^{2}$ In their native form the disaccharides contain a glucosamine

dure this constituent is converted to ${ }^{3} \mathrm{H}$-anhydromannitol. Since direct nitrous acid treatment released about $90 \%$ of the two disulphated disaccharides, it would appear that their glucosamine residues are present in the $N$-sulphated form.

${ }^{3}$ Values are presented as the molar per cent (means \pm SEM) of the total sulphated disaccharides. The sulphated chondroitin disaccharides containing anhydrotalitol (originating from galactosamine) which represented only $4 \%$ and $6 \%$ of the total disaccharides in the nondiabetic and diabetic samples, respectively $(p>0.05)$, were not included in this summation.

${ }^{4} \mathrm{NS}=$ not significant $(p>0.05)$.
\end{abstract} residue and after the hydrazine/nitrous acid/ $\mathrm{NaB}\left[{ }^{3} \mathrm{H}\right]_{4}$ proce-

betic GBM indicated that only one species, namely $\operatorname{IdUA}(2 \mathrm{~S}) \alpha 1 \rightarrow 4 \mathrm{AnManH}_{2}(3 \mathrm{~S})$, originating from the $\operatorname{IdUA}(2 \mathrm{~S}) \alpha 1 \rightarrow 4 \mathrm{GlcNS}(3 \mathrm{~S})$ sequence was significantly different (Table 1). This unusual disulphated species which had previously been observed in the bovine GBM heparan sulphate [5] occurred in the diabetic samples in less than half the amount of that in the nondiabetic GBM and this was clearly evident when the values from individual cases were plotted (Fig. 1).

\section{Discussion}

While this study has confirmed the previously reported decrease of heparan sulphate proteoglycan in the diabetic GBM, it has more importantly uncovered a well-defined structural alteration in the saccharide chains. The highly specific reduction in $\operatorname{IdUA}(2 \mathrm{~S}) \alpha 1 \rightarrow 4 \mathrm{GlcN}(3 \mathrm{~S})$ presents an intriguing finding, as this disaccharide unit has so far been found only in the heparan sulphate from GBM. Indeed, this unusual disaccharide component was found to represent a major species in the human GBM as was noted in the GBM from bovine kidneys [5]. The presence of a 3-O-sulphated glucosamine residue is by itself a rare feature in heparan sulphates from other sources and is a distinguishing component of the antithrombin-binding region of these polysaccharide chains where it invariably occurs in a pentasaccharide sequence containing GlcUA $\beta 1 \rightarrow 4 \mathrm{GlcNS}(3 \mathrm{~S} \pm 6 \mathrm{~S})$ [6, 7]. This disaccharide could not be detected in bovine GBM [5] nor could we find it in the present study on the human material. The 3-O-sulphation of glucosamine residues is considered to be the final even in a highly ordered series of biosynthetic steps by which the repeating GlcUA $\beta 1 \rightarrow 4$ GlcNAc units of the heparan sulphate polymer are modified to yield the mature molecule [7]. Whereas initial investigations indicated that the 3-O-sulphotransferase acts specifically on glucosamine residues within the antithrombin binding sites (i.e. on the GlcUA $\beta 1 \rightarrow$ $4 \mathrm{GlcNS} \pm 6 \mathrm{~S}$ disaccharide unit), recent studies have indicated that isoforms of that enzyme (3-OST- $\left.3_{\mathrm{A}, \mathrm{B}}\right)$ transfer sulphate to $\operatorname{IdUA}(2 \mathrm{~S}) \alpha 1 \rightarrow 4 \mathrm{GlcNS}$ sequence [8]. Since it is this unusual disaccharide unit which we find in the GBM, it is likely that these newly discovered enzyme variants are involved in the 3-O-sulphation of its glucosamine residue. In view of the relatively high content of the IdUA(2S) $\alpha 1 \rightarrow 4$ GlcNS(3S) disaccharide unit in GBM (19\% of sulphated disaccharides in nondiabetic GBM) the amount of this enzyme in normal glomerular cells must be substantial. Indeed the isoforms 3OST- $3_{\mathrm{A}}$ and $3-\mathrm{OST}-3_{\mathrm{B}}$ have been observed to be well-expressed in human kidneys [8]; furthermore, the $\operatorname{IdUA}(2 \mathrm{~S}) \alpha 1 \rightarrow 4 \mathrm{GlcN}(3 \mathrm{~S})$ sequence is produced by glomerular cells in culture [9]. In view of the specificity of the structural alteration observed in the heparan sulphate from human diabetic GBM, it is reasonable to ascribe the decreased content of the $\operatorname{IdUA}(2 \mathrm{~S}) \alpha 1 \rightarrow 4 \mathrm{GlcN}(3 \mathrm{~S})$ disaccharide to a lower activity of the $3-\mathrm{OST}-3_{\mathrm{A}, \mathrm{B}}$ enzyme in the glomerular cells of diabetic kidneys.

Whereas the reduced amount of this disulphated disaccharide, in addition to the decreased content of the entire heparan sulphate proteoglycan molecule, could contribute to an impairment in the glomerular anionic filtration barrier, it seems more likely that the loss of a sulphate group at a defined location along the heparan sulphate chains would have a more subtle biological consequence. 


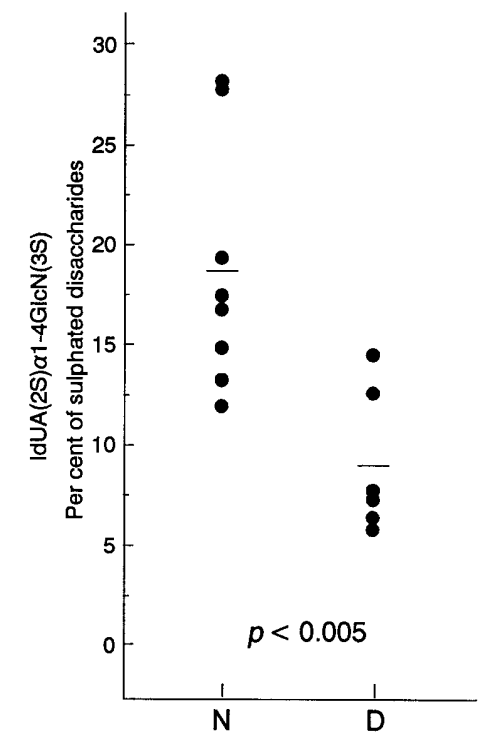

Fig. 1. Comparison of the $\operatorname{IdUA}(2 \mathrm{~S}) \alpha 1 \rightarrow 4 \mathrm{GlcN}(3 \mathrm{~S})$ content of the heparan sulphate from GBM of nondiabetic $(N)$ and diabetic $(D)$ human subjects. This disaccharide unit is obtained as $\operatorname{IdUA}(2 \mathrm{~S}) \alpha 1 \rightarrow 4 \mathrm{AnManH}_{2}(3 \mathrm{~S})$ after hydrazine/nitrous acid/ $\mathrm{NaB}\left[{ }^{3} \mathrm{H}_{4}\right]$ treatment (see Table 1). The values are expressed as per cent of total sulphated disaccharides

It has become apparent that the heparan sulphate chains are involved in a selective manner in interactions with various biologically important proteins $[6$, 7]. The multiple modifications which the polymer could undergo by such events as site-specific sulphate transfers and glucuronic acid C-5 epimerisation can lead to protein-binding domains among which the antithrombin-binding region represents the most welldefined example. Other proteins which have been noted to bind to heparan sulphate include interleukin, fibroblast growth factor, vascular endothelial growth factor, lipoprotein lipase, interferon- $\gamma$ and platelet factor $4[6,7]$. It has most recently been reported that the herpes simplex virus 1 can gain entry into cells by binding to a site which has been modified to include the $\operatorname{IdUA}(2 \mathrm{~S}) \alpha 1 \rightarrow 4 \mathrm{GlcN}(3 \mathrm{~S})$ sequence [10].

Although it would appear that this disaccharide unit is not involved in anticoagulant activity [8], the possibility that in the GBM it is the determining part of a domain which interacts with circulating proteins, angiogenesis factors, cell adhesion molecules or other GBM components merits exploration.

Acknowledgements. This work was supported by Grant DK 17325 (to R. G. Spiro) from the National Institutes of Health and by a grant as well as a Career Development Award (to A.S. B. Edge) from the Juvenile Diabetes Foundation.

\section{References}

1. Sternberg M, Cohen-Forterre L, Peyroux J (1985) Connective tissue in diabetes mellitus: biochemical alterations of the intercellular matrix with special reference to proteoglycans, collagens and basement membranes. Diabetes Metab 11: $27-50$

2. Spiro RG (1988) Pathogenesis of diabetic glomerulopathy: a biochemical view. In: Mogensen CE (ed) The Kidney and Hypertension in Diabetes Mellitus. Martinius Nijhoff, Boston, pp 117-130

3. Kofoed-Enevoldsen A (1995) Heparan sulphate in the pathogenesis of diabetic nephropathy. Diabetes Metab Rev 11: 137-160

4. Shimomura H, Spiro RG (1987) Studies on the macromolecular components of human glomerular basement membrane and alterations in diabetes. Decreased levels of heparan sulphate proteoglycan and laminin. Diabetes 36: 374-381

5. Edge AS, Spiro RG (1990) Characterization of novel sequences containing 3-O-sulphated glucosamine in glomerular basement membrane heparan sulphate and localization of sulphated disaccharides to a peripheral domain. J Biol Chem 265: 15874-15881

6. Rosenberg RD, Shworak NW, Liu J, Schwartz JJ, Zhang L (1997) Heparan sulphate proteoglycans of the cardiovascular system. Specific structures emerge but how is synthesis regulated? J Clin Invest 99: 2062-2070

7. Lindahl U, Kusche-Gullberg M, Kjellén L (1998) Regulated diversity of heparan sulfate. J Biol Chem 273: 24979-24982

8. Liu J, Shworak NW, Sinaÿ P et al. (1999) Expression of heparan sulphate D-glucosaminyl 3-O-sulphotransferase isoforms reveals novel substrate specificities. J Biol Chem 274: 5185-5192

9. Shen G-Q, Kresbach G, Spiro MJ, Spiro RG (1995) Evaluation of the cell specificity and sulfate dependence of glomerular extracellular matrix proteoglycan synthesis. Arch Biochem Biophys 321: 83-93

10. Shukla D, Liu J, Blaiklock P et al. (1999) A novel role for 3-O-sulphated heparan sulphate in herpes simplex virus 1 entry. Cell 99: 13-22 